\title{
La intangibilidad de las cooperativas como ventaja competitiva
}

\author{
Ignacio Ruiz-Guerra ${ }^{\star}$, Valentín Molina-Moreno ${ }^{\star *}$
}

* Doctor en Económicas, Universidad de Granada. Profesor asociado de Organización de Empresas, Universidad Complutense de Madrid, Madrid, España.

Correo electrónico: ignacio.ruiz@ccee.ucm.es

** Doctorado en Ciencias Económicas y Empresariales, Universidad de Castilla-La Mancha. Profesor, Universidad de Granada, Granada, España.

Correo electrónico: valentinugr1@gmail.com

Recibido: 5 de septiembre del 2014 Aprobado: 7 de noviembre del 2014

Cómo citar este artículo: Ruiz-Guerra, I. y Molina-Moreno, V. (2014). La intangibilidad de las cooperativas como ventaja competitiva. Cooperativismo \& Desarrollo, 22(105), 47-54. doi: http://dx.doi.org/10.16925/ co.v22i105.1034

\begin{abstract}
Resumen
El éxito competitivo de las empresas proviene, como ya anunciaban Navas y Urbina (2002), de los activos intangibles por la identificación y medición, lo cual ha resultado ser imprescindible para las organizaciones a fin de desarrollar una evaluación correcta de sus procesos productivos. La valorización de los procesos productivos y del área de gestión de las cooperativas no se hace ajeno a este proceder, ni reciben el valor que merecen, ya que tampoco el sector cooperativo ha valorizado su posicionamiento en el entorno productivo en el que se mueve y lo que ello conlleva. Sin embargo, hoy en día las estrategias de las empresas y de las cooperativas precisan de un correcto y acertado conocimiento de la intangibilidad de los propios productos, y de lo que conlleva la actividad de las cooperativas en los entornos en los que se mueven. Los activos intangibles se están convirtiendo en la clave del éxito competitivo de las empresas, por lo que su identificación, medición y evaluación es un estudio relevante e, incluso ahora, imprescindible para las organizaciones. En este artículo se han elegido las cooperativas de la provincia de Toledo como ejemplo de potencialidad de aplicación de estrategias de valoración de intangibles, en tanto herramienta de ventaja competitiva ante la apertura de nuevos canales de comercialización internacionales.
\end{abstract}

Palabras clave: cooperativas, intangibilidad, rentabilidad, valores, ventaja competitiva.

\section{The Intangibility of Cooperatives as a Competitive Advantage}

\begin{abstract}
The competitive success of companies, according to Navas and Urbina (2002), comes from the identification and management of intangible assets, which has become indispensable for organizations when making a correct assessment of their productive processes. The valuation of productive processes in the field of cooperative management has not remained aloof from this procedure, but nor does it receive the value that it deserves, because the cooperative sector has also failed to value its positioning in the productive realm in which it works and what this leads to. However, the strategies of companies and cooperatives today require a correct and sure knowledge of the intangibility of their own products, and of the effects of their activities in the areas in which they move. Intangible assets are becoming the key to the competitive success of companies, so that their identification, measurement and assessment is a relevant field of study that is now even indispensable for an organization. In this article, the cooperatives in the province of Toledo have been chosen as an example of the potential for the application of strategies for valuation of intangibles, and as a tool for competitive advantage in the context of the opening of new international commercialization channels.
\end{abstract}

Keywords: cooperatives, intangibility, profitability, values, competitive advantage.

\section{A intangibilidade das cooperativas como vantagem competitiva}

\section{Resumo}

O sucesso competitivo das empresas provém, como já anunciavam Navas e Urbina (2002), dos ativos intangíveis pela identificação e medição, o que resulta ser imprescindível para as organizações a fim de desenvolverem uma correta avaliação de seus processos produtivos. A valorização dos processos produtivos e da área de gestão das cooperativas não é alheia a esse proceder nem recebe o valor que merece, já que o setor cooperativo também não tem valorizado seu posicionamento no contexto produtivo no qual se move e o que isso implica. Contudo, hoje em dia, as estratégias das empresas e das cooperativas precisam de um adequado conhecimento da intangibilidade dos próprios produtos e do que implica a atividade das cooperativas nos ambientes nos quais atuam. Os ativos intangíveis estão se convertendo na chave do sucesso competitivo das empresas, portanto o estudo de sua identificação, medição e avaliação torna-se relevante e, atualmente, imprescindível para as organizações. Neste artigo, foram escolhidas as cooperativas da província de Toledo como exemplo de potencialidade de aplicação de estratégias de valoração de intangíveis enquanto ferramenta de vantagem competitiva ante a abertura de novos canais de comercialização internacionais.

Palavras-chave: cooperativas, intangibilidade, rentabilidade, valores, vantagem competitiva. 


\section{Introducción}

En un mundo globalizado, la competitividad de las empresas, sectores y territorios depende de la capacidad de innovación (Organización de Cooperación y Desarrollo Económico [OCDE], 1999). La innovación comporta un proceso complejo y colectivo en el que los ámbitos rutinarios de la empresa (producción, comercialización, etc.) han adquirido una más que apreciable relevancia. La innovación es entendida como un proceso en el que con frecuencia es necesaria la participación de una pluralidad de actores distintos, los cuales interaccionarían definiendo sistemas de innovación (Lundvall y Johnson, 1994).

Ante esto, además, el territorio puede propiciar una proximidad geográfica entre actores (Rallet y Torre, 2004), sobre todo unos valores y unas representaciones compartidas que favorezcan la generación de confianza entre dichos actores y, por consiguiente, la cooperación entre estos necesaria para generar innovaciones.

El sistema agroalimentario español y, en particular, el sistema agroalimentario de Castilla-La Mancha están centrados mayoritariamente en el sector vitivinícola y el sector oleícola, destacando sobre el resto. Estos sectores necesitan innovar con el fin de competir, tal como se ha hecho evidente en el momento en el que los mercados se han paralizado por causa de la crisis económica mundial en la que vivimos - la primera crisis global-, siendo estos dos sectores los que más han sufrido la paralización de los mercados por la ralentización del consumo por parte de los clientes. A su vez, han tenido que crear mecanismos de cooperación para el mantenimiento y supervivencia de los sectores agrícolas en el medio rural, ya fuera de manera formal o informal (Gallego, 2008), entre empresas y otros actores (públicos o privados), los cuales han sido necesarios a fin de que estos sistemas operen como auténticos sistemas de innovación y no solo de producción en un momento de tanta incertidumbre (Cooke, Uranga y Etxebarría, 1998).

Las cooperativas desarrollan su actividad económica de acuerdo con unos principios que pueden alimentar el crecimiento de una trama de relaciones socioeconómicas (capital relacional) y sociopolíticas (capital social) propicias, así como para generar la confianza que requiere la cooperación entre empresas y entre estas y el sistema científico-tecnológico. Así, se podría facilitar la creación de la proximidad organizada (relacional) necesaria para dotar de fluidez a las relaciones entre actores distintos dentro de los sistemas territoriales de producción y de innovación. Sin embargo, la presencia de una amplia red de cooperativas también podría definir ciertas inercias y factores de bloqueo.

Este artículo profundiza en las estrategias que potencialmente puede explotar el sector oleícola (aceite de oliva), y cuyos resultados estarían significativamente condicionados por la presencia de las cooperativas almazaras y las innovaciones que se llevaran a cabo en ellas.

Las cooperativas son ricas en capital social (Spear, 2001; Morales, 2002; Mugarra, 2005). Por tanto, en la medida que el capital social favorece la confianza y la cooperación entre actores, las cooperativas se erigen en estímulo al desarrollo territorial (Mozas y Bernal, 2006), y de esta manera a la supervivencia del entorno en el que llevan a cabo su actividad. Partiendo de la base de que las cooperativas forman parte del entramado institucional que constituye la matriz comportamental de estos sistemas, resulta lógico inferir que van a influir en la naturaleza y evolución del proceso innovador. Las cooperativas pueden ser esenciales en el estadio interrelacional de las empresas.

\section{Dirección estratégica de la innovación}

Autores especializados en el sector agroalimentario han afirmado de unos años a esta parte cómo el caso concreto del sector cooperativo carece de un sistema de estrategias "en aval" por parte de las cooperativas, excepto algunas experiencias más modernas (Sanz, Rodríguez-Zúñiga y Mili, 1998). Esto viene dado por las cooperativas que no están preparadas para el desarrollo de un modelo de comercialización eficiente y competitivo.

Las administraciones autonómicas y locales son las que tienen asignado un papel más relevante en el fomento de las etapas de la innovación más cercanas al mercado (Mulet, 1999). Por el contrario, la administración estatal tiene una responsabilidad normativa y de coordinación, y de velar por la creación de conocimiento científico.

La innovación es otra de las estrategias que han seguido según las necesidades, o las capacidades de cada una de las cooperativas, pues son las que concentran la mayor parte de la producción: "innovar es convertir invenciones en procesos, productos o servicios que tienen éxito en el mercado. Sin consecuencias 
positivas sobre la generación de beneficios no hay innovación" (Mulet, 1999).

Esta aplicación conceptual de la innovación está generando ciertas descompensaciones en muchas cooperativas, las cuales han realizado importantes inversiones en tecnología y no están recibiendo unos rendimientos acordes con las necesidades financieras que las acucian.

Para Mulet (1999), existen tres tipos de innovación atendiendo al origen de la idea de partida:

- Gerenciales

- Comerciales

- Tecnológicas

Las bajas capacidades tecnológicas y de competitividad se deben, no solo a factores parcialmente estáticos como pueden ser la carencia de infraestructuras adecuadas o a la falta de instrucciones, sino también al déficit de capital social (Coronado y Acosta, 1999), que adolecen las cooperativas almazaras para afrontar un desarrollo tecnológico que les haga estar a la última en avances. Esto no significa que los adelantos alcanzados hasta el día de hoy no hayan sido importantes, sino que en ciertos lugares tienden a ser insuficientes. Es necesario, por lo tanto, dar un giro de una estrategia de oferta fundamentada en proporcionar recursos para desarrollar actividades de I+D+i, hacia una de demanda en la que el cooperativismo tenga un papel más activo.

El concepto de innovación en el sector cooperativo se basa también en un detalle de "saber-hacer" localista, el cual a su vez tiene como fundamento una transferencia o integración técnica que concierne a la transmisión de la tecnología en el seno de las empresas-cooperativas de pequeño tamaño. No obstante, ese saber-hacer también se ha dejado con un "dejar-hacer" de las cooperativas con respecto a los intermediarios, siendo ellos los que han provocado grandes lastres al sector, de manera que se profundizan las carencias de este por su inmovilismo de muchos años en la búsqueda de nuevas soluciones de comercialización.

En este caso, las cooperativas han evolucionado desde la entrada en la Unión Europea, al cambiar el sistema de los procesos productivos con una clara ventaja comparativa en la transmisión del "saber-hacer" local como valor añadido a las mejoras e innovaciones tecnológicas. Es decir, si bien los avances técnicos han ayudado a mejorar la calidad de los productos agroalimentrios y han estandarizado los métodos de producción, el sector - como recoge este estudio- tiene un algo más, ese valor añadido que lo hace ser imprescindible en los hogares en la cultura mediterránea. La innovación tecnológica es una ventaja y un beneficio, pero sin olvidar el verdadero valor intrínseco del producto que se está tratando: "cuanto mayor sea la importancia del cambio en el entorno, más debe procurarse basar la estrategia en sus recursos y capacidades internas, en lugar de hacerlo en el mercado" (Grant, 1996).

\section{Diferenciación competitiva del producto}

Considerar los recursos y las capacidades internos como elementos estratégicos exige que estos sean escasos, valiosos y difícilmente imitables y sustituibles (Barney, 1991). Es necesario que el análisis interno de las competencias fundamentales se torne en elemento básico e ineludible en la formulación de la estrategia del sector (Hamel y Prahalad, 1990).

Cuando se pretende la comercialización de productos agroalimentarios se busca obtener ventajas mediante la diferenciación de productos, de manera que tendrá que competir básicamente con el concepto de calidad de producto. De unos años a esta parte se ha pasado de un consumo de masas a un consumo de calidad, en el cual el consumidor no se rige tanto por el precio sino por la calidad de los productos (Sanz et al., 1998). Los pilares fundamentales sobre los que se había regido la diferenciación de los productos podía venir condicionada por diferentes alternativas (Porter, 1980):

- Calidad del producto

- Confianza en el producto

- Innovación de producto

- Servicios periféricos

- Marca

La diferenciación a través de la calidad ha sido la apuesta del sector cooperativo, lo cual permitirá vender a precios más altos y, por tanto, conceder mayor rentabilidad a los cooperativistas. Este concepto de calidad se certifica a través de organismos tales como las denominaciones de origen (DO), las indicaciones geográficas protegidas (IGP), o la agricultura ecológica (Ecolabel).

Esto es así si se tiene en cuenta que pertenece a un sistema económico integrado por trabajadores y organizaciones en el que el conocimiento está basado en la producción y consumo de saberes, y en el cual los productos no son un conjunto de objetos físicos sino más bien una variedad de servicios intangibles por la incorporación 
del conocimiento al activo que supone el producto (Bueno, 2000a). Las estrategias de internacionalización, la diferenciación y la diversificación hacia nuevas gamas de productos $-\mathrm{y}$ ante la necesidad de reducir costes y mejorar la competitividad la racionalización de los procesos logísticos- están adquiriendo especial relevancia en las estrategias empresariales.

Entre estas estrategias también se encuentran las nuevas formas de acuerdo y relaciones entre los productores y los canales de distribución agroalimentaria, principales proveedores de las crecientes grandes superficies que, desde hace unos años, se han convertido en los principales vendedores en nuestro país. Suponen 54,4\% (Porter, 1980; Sanz, Rodríguez-Zúñiga y Mili, 1997), un porcentaje más alto - solo en este producto- que la media de ventas totales en cuanto a productos alimentarios del que se encargan de la comercialización de $45 \%$. Esto quiere decir que los clientes tienden a proveerse de este producto en este tipo de superficies más que de otros productos también alimentarios. Esta competencia horizontal desarrollada en los últimos años ha provocado que los márgenes de beneficio se hayan ajustado considerablemente, reduciendo las vías para obtener crecimientos presupuestarios.

Otro tipo de estrategias que se siguen en los mercados agroalimentarios - en cuanto se pretende abrir nuevos canales de comercialización- parten de la base no solo de los estudios de mercado, en los que las cifras van a dar una información teórica de lo que realmente puede funcionar. En este caso, lo que importa es que el producto sea adecuado para el gusto del consumidor, por lo que se realizan estudios con consumidores de diferentes productos con catas a ciegas, lo cual permite conocer sus gustos, si los sabores se adecuan a su paladar, si los olores serán adecuados y atractivos para el consumidor, etc.

La estrategia por la que el sector oleícola aún no ha apostado - aunque viene siendo la causa primordial del aumento de la demanda de productos agroalimentarios de calidad a nivel mundial- es su carácter diferenciado. Es notoriamente conocido que los productos agroalimentarios de España son reconocidos internacionalmente, ya que solo pueden ser producidos en lugares con unas características climatológicas determinadas, y la producción suele tener diferencias en cuanto a volumen dependiendo del año al que nos refiramos. La cotidianeidad de esta clase de productos, como decíamos al principio, provoca que no se valore lo intangible, es decir, lo que no se puede tocar, lo que se da por visto o satisfecho, como algo natural, pero que es fruto del paso del tiempo y de la evolución de la sociedad.

Este tipo de diferencias suponen que el producto tenga un arraigo popular y social mucho mayor que el del resto de sus competidores en el mercado. La clara diferenciación en el mercado, si se hace visible y se valoriza, provocará una revalorización competitiva del sector y de este producto en particular, haciendo ver que, en muchos casos, la diferencia de precio está justificada por el mayor impacto que tiene en la sociedad uno con respecto a los otros.

\section{Intangibles del producto agroalimentario}

En la sección anterior, hemos resaltado la estrategia que se podría proporcionar a aquellos intangibles de los productos agroalimentarios, los cuales suponen un activo para el producto y le proporcionan una diferenciación competitiva con respecto al resto de productos que le son competencia. Pero para obtener la directriz de intangibilidad se ha partido de la definición de intangible para luego aplicársela al producto final.

Una primera clasificación sobre los diferentes intangibles que se encuentran en la literatura separa a los activos intangibles de acuerdo con cuatro criterios:

- Origen. En función del origen de su naturaleza, distinguiremos entre recursos humanos y no humanos. Dentro de los recursos no humanos, podemos distinguir recursos tecnológicos, comerciales y organizativos.

- Separables del individuo que los creó. En función de la posibilidad que al activo se lo pueda separar o no del individuo o individuos que los crearon.

- Defensa legal. En función de la posibilidad de defensa legal ante el uso exclusivo del bien.

- Transparencia de la información. Este aspecto está relacionado con el grado de transparencia u opacidad de la información sobre la que se basan los recursos (Vargas-Montoya, 2000).

Según Bueno (2000b):

El capital intangible es el conjunto de competencias básicas distintivas de carácter intangible que permiten crear y sostener la ventaja competitiva; a su vez son el conjunto de actitudes, conocimientos implícitos y explícitos y de capacidades de carácter personal, tecnológico y organizativo. 


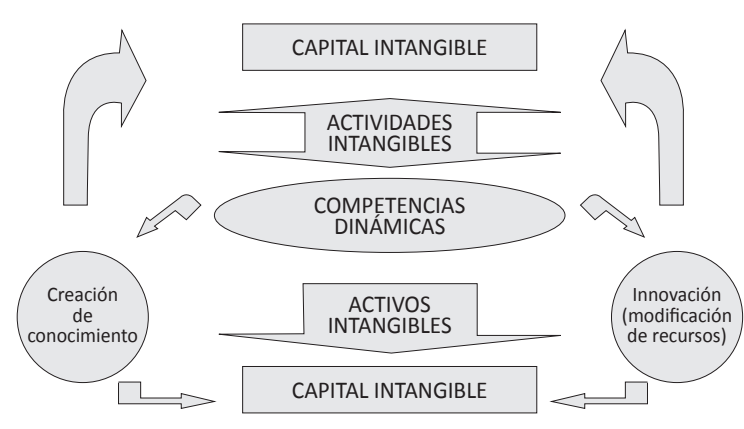

Figura 1. Diagrama del capital intangible. Tomado de "La sociedad del conocimiento reclama capital intangible”, por E. Bueno, $2000 \mathrm{~b}$.

Los intangibles tenderán a convertirse en recursos ganadores de riqueza, tanto para las empresas como desde una perspectiva más amplia (Nonaka, 1991; Cooke, 2002; Bontis, 2003). La acertada división entre capital tangible físico-financiero y capital intangible permite hacerse una idea del marco que recoge este tipo de afirmaciones en el sistema de intangibilidad.

Estos elementos intangibles del aceite de oliva se pueden dividir en los siguientes puntos. Haciendo un brindis al sector, se les denominará bajo la certidumbre de la pertenencia etimológica al sector oleícola:

\section{Cultura}

- Historia

- Origen

- Significado religioso

- Arqueología

- Literatura

- Trascendencia en obras universales

- Cánticos populares

- Poesía popular y refranero español

- Pintura

- Protagonismo en obras de artistas de renombre

\section{Socioeconomía}

- Superficie

- Producción

- PAC

- Salud

- Cosmetología

- Prevención enfermedades

- Dieta mediterránea

\section{Economía}

- Exportación

- Importación

- Industria especializada

- Empresas (cooperativas, industriales)

- Envasadoras

- Entramadoras

- Mercado de futuros

- Arraigo población al medio rural

- Empleo del sector

- Ecología: efectos al medioambiente

- Derechos de $\mathrm{CO}_{2}$

- Contra la desertización

- Turismo: práctica turística para conocer el proceso de cultivo, producción y características

Las aproximaciones de los últimos años al concepto de intangible, además de enfatizar en la inmaterialidad de su naturaleza (Itami y Roelh, 1987), establecen que los activos intangibles se identifican genéricamente con servicios productivos proporcionados por el capital humano, tecnológico y comercial, así como por el conjunto de todos ellos. Sin embargo, a la hora de clasificar y definir los recursos, la literatura es profusa y los criterios subyacentes en cada autor son de lo más heterogéneo (Hall, 1992; Markides y Williamson, 1994; Miller y Shamsie, 1996; Grant, 1996; Suárez, 1999; Calvo y López, 2003). De acuerdo con Hall, "los recursos intangibles deben ser considerados como activos porque son cosas poseídas, muchas de las cuales a menudo tienen protección legal" (1992, p. 13). En este caso, no tienen protección legal, sino que significan en muchos de los casos aplicados, a los que se refieren a diferentes actos, bienes e incluso obras que son patrimonio, bien de la humanidad, o bien inmaterial de la sociedad de la que procede.

\section{La internacionalización de lo agroalimentario}

Desde el punto de vista comercial, las cooperativas realizan, fundamentalmente, una labor de producción y almacenamiento. Del total de su producción, una pequeña parte la venden envasada, aunque la mayor parte de la producción es vendida a granel a las industrias 
de refinado o envasadoras. La escasa implicación de los productores en la comercialización de sus productos, perdiendo con ello el valor añadido que generan las actividades de comercialización, se revela entre las causas más importantes que en la literatura se apuntan como explicativas de esta situación (Mozas, Murgado, Parras, Senise y Torres, 1997):

- Los socios cooperativistas no se han preocupado por la calidad.

- Falta de orientación de las cooperativas hacia el mercado final.

- Falta de profesionalización del sector cooperativo y, por lo tanto, escasa orientación al mercado y ausencia de gestión empresarial.

- Reticencia a la realización de inversiones en actividades comerciales.

El producto agroalimentario cooperativo ha de mejorar las estrategias de competitividad a fin de entra con éxito en nuevos mercados. Como una estrategia importante, destaca el uso razonable de su estrategia competitiva de base, que es la clave para el éxito de las empresas. Debido a que varias diferencias en la estrategia básica resultan muy grandes en las empresas, es necesario implementar con éxito sus necesidades legítimas con los diferentes recursos y habilidades. La estrategia básica también significa medidas de organización, procedimientos de control e innovadoras diferencias institucionales.

Las empresas deben tomar en consideración lo que significa la estrategia competitiva de base, la cual generalmente se basa en sus necesidades únicas para determinar el estado de los recursos y, de esta manera, tomar las estrategias de comercialización apropiadas. En las primeras etapas de las empresas, cuando se busca entrar en un sector y obtener un punto de apoyo, es muy importante. Es posible que se adopte la estrategia competitiva de base, como un factor común, dado el limitado número de recursos que se utiliza en un mercado tan particular como el agroalimentario cooperativo, asentando así un determinado segmento en el mercado de la industria. Sin embargo, cuando acapare la suficiente fuerza, es posible que no se conformen con ser un líder en un segmento concreto del mercado; es en este momento cuando pueden recurrir a ajustar su estrategia de base a la diferenciación total a través de la estrategia de liderazgo en costes, a fin de alcanzar una mayor cuota de mercado y así eventualmente reemplazar al líder original que existiera en el sector oleícola y convertirse en el líder del mercado sectorial.
Si el sector cooperativo no logra identificarse con su propia estrategia competitiva básica, entonces se ve atrapado en la posición central, por lo tanto queda en una situación estratégica de negocio en condiciones muy desfavorables, careciendo de una cuota de mercado, y no alcanza un marco de diferenciación de los productos teniendo que tender a la ventaja de costes de recolección. En la industria cooperativa agroalimentaria, muchas empresas carecen de una buena situación por sus propios recursos y se encuentran atrapados entre unas estrategias en las que los beneficios son escasos e incluso son incapaces de mantener su posición en el mercado al que han accedido. Algunas de estas empresas, al no haber detectado de manera temprana su ventaja competitiva, tienen que tender a establecer una estrategia competitiva de base. Ya sea para tomar las medidas necesarias para alcanzar el liderazgo en costes, o al menos un nivel de costes comparable con los que tiene la competencia, necesitará de una inversión de marketing positivo para alcanzar la magnitud de la contratación y, por lo tanto, la necesidad de alcanzar la cuota de mercado mínima.

Es necesario que la capacidad de la empresa dentro de la industria del aceite de oliva logre a largo plazo un importante nivel de desarrollo, en el cual la construcción de marca corporativa sea un pilar fundamental a nivel estratégico para la implantación comercial del sector cooperativo en el mercado nuevo.

\section{Conclusiones}

El sector cooperativo agroalimentario necesita de la innovación para llevar a cabo un novedoso sistema de comercialización que le genere una seguridad de venta de productos, con un valor añadido que, hasta ahora, no ha sabido explotar por la condición asumida en el tiempo. Esto en cuanto la cooperativa solo se dedica al almacenamiento de la producción oleícola, y había que esperar a que los intermediarios se encargaran de la comercialización, que es, en definitiva, donde se alcanzan los más importantes beneficios en el sector agroalimentario.

Del mismo modo, las necesidades mostradas en el artículo hacen que sea imperiosa en las cooperativas la creación de nuevos cuadros de mando que conozcan el funcionamiento de los mercados y sepan de gestión administrativa y empresarial, y estén especializados en el particular funcionamiento y gestión de las cooperativas agroalimentarias, y las almazaras en particular. 
El comercio exterior es la solución para asegurar la comercialización de la producción de las cooperativas, pero si se tiene en cuenta que las estrategias que han llevado a cabo hasta ahora han partido de la base del inmovilismo, es adecuado pensar que los claros déficits de conocimiento y de experiencia en el comercio exterior puedan llevar al fracaso. Para ello, las estrategias determinan que el sector cooperativo, conservador e inmovilista nunca tenderá al riesgo excesivo en la búsqueda de nuevas soluciones, pero es necesario el cambio en la situación económica actual.

El cambio se ha producido ya con la generación de nuevos tipos de alianzas y cooperación intercooperativa para la creación de mayor representatividad en los mercados, con unos volúmenes más importantes de producción que permitan una mayor flexibilidad en la venta de la producción a las industrias refinadorasenvasadoras, o bien la venta directa en los mercados internacionales.

También se hace necesaria la actuación de las autoridades educativas y culturales, con el objetivo de no dejar que se pierda una parte fundamental de nuestro patrimonio: el patrimonio intangible que supone la pertenencia a una cultura determinada, con unos rasgos diferenciados y una serie de productos característicos que trascienden de lo meramente agrícola para ser un elemento vertebrador dentro de la cultura, la sociedad y la tradición que representa.

Una vez que las autoridades muestren el verdadero significado de los productos agroalimentarios cooperativistas y, sobre todo, la sociedad de consumidores tenga más en cuenta esa caracterización específica con respecto a ellos, tenderá a ver en el producto una serie de ventajas competitivas más allá de lo tangible en el mercado: el precio.

Una vez que esto repunte, y así se está viendo en los márgenes evolutivos macroeconómicos, la sociedad mundial comprende que el consumo de productos agroalimentarios de calidad - los cuales provienen de una explotación cooperativa- conlleva beneficios para el entorno donde se lleva a cabo la actividad. Ese incremento de demanda ha ayudado a que la oferta también crezca con inversiones en nuevas superficies de cultivo, pero esto tiene que tender, también, a la consecución, por parte de los productores primigenios (los cooperativistas), de una repercusión en los precios que reciben de la venta de sus productos, y tender a conseguir mayor parte del valor añadido que están disfrutando los intermediarios por desconocimiento de las estrategias de valorización de su producto.

\section{Referencias}

Barney, J. B. (1991). Firm Resources and Sustained Competitive Advantage. Journal of Management, 17, 99-120.

Bontis, N. (2003). National Intellectual Capital Index. UNDP/ RBAS (Working paper).

Bueno, E. (2000a). La economía del conocimiento. La importancia de los intangibles. En E. Bueno y M. P. Salvador, Perspectivas sobre dirección de conocimiento y capital intelectual. Madrid: Euroforum Escorial.

Bueno, E. (2000b). La sociedad del conocimiento reclama capital intangible. En E. Bueno, A. Aragón, García-Morales, El capital intangible frente al capital intelectual de la empresa desde la perspectiva de las capacidades dinámicas. (Documento de trabajo). Zaragoza, XI Congreso Nacional de ACEDE.

Calvo, A. y López, V. (2003). Percepción de intangibles y sus consecuencias sobre el rendimiento empresarial: una aproximación empírica a las PYMEs gallegas. Revista Galega de Economía, 12(2), 1-16.

Cooke, P., Uranga, M. C. y Etxebarría, G. (1998). Regional Systems of Innovation: An Evolutionary Perspective. Environment and Planning A, 30, 1563-1584.

Cooke, P. (2002). Knowledge Economies: Clusters, Learning and Cooperative Advantage. London: Routledge.

Coronado, D. y Acosta, M. (1999). Innovación tecnológica y desarrollo regional. Información Española, 781, 103116.

Gallego, J. R. (2008). La articulación local-global de sistemas territoriales de producción y de innovación. Revista de Estudios Regionales, 84, 53-82.

Grant, R. (1996). Dirección estratégica. Madrid: Civitas.

Itami, H. y Roelh, T. (1987). Mobilizing Invisible Assets. Cambridge: Harvard University Press.

Hamel, G. y Prahalad, C. K. (1990). The Core Competence of the Corporation. Harvard Business Review, 68, 79-91.

Hall, R. (1992). The Strategic Analysis of Intangible Resources. Strategic Management Journal, 13(2), 135-144.

Lundvall, B. A. y Johnson, B. (1994). The Learning Economy. Journal of Industry Studies, 1(2), 23-42.

Markides, C. y Williamson, P. (1994). Related Diversification, Core Competencies and Corporate Performance. Strategic Management Journal, 15, 149-167.

Miller, D. y Shamsie, J. (1996). The Resource-Based View of the Firm in Two Enviroments: the Hollywood Films Studios from 1936 to 1965. Academy of Management Journal, 39, 519-543.

Morales, A. C. (2002). La construcción del capital social a través de la economía social: el caso andaluz. REVESCO, 78, 85-119. 
Mozas, A. y Bernal, E. (2006). Desarrollo territorial y economía social. CIRIEC-España, 55, 125-140.

Mozas, A., Murgado, E., Parras, M., Senise, O. y Torres, J. (1997). La concentración de las cooperativas oleícolas en Andalucía y sus efectos en la comercialización de aceites envasados. En Estudios socio-económicos y de mercado encaminados a la concentración de la oferta por parte de las cooperativas. Universidad de Jaén.

Mugarra, A. (2005). Capital social y cooperativas: la experiencia en el país vasco. Economiaz, 59, 307-329.

Mulet, J. (1998). La innovación tecnológica, fuente de competitividad para la empresa española. Economistas, 17(80), 356-369.

Navas, J. E. y Ortiz de Urbina, M. (2002). El capital intelectual en la empresa: análisis de criterios y clasificación multidimensional. Economía Industrial, 346, 163-172.

Nonaka, I. (1991). The Knowledge Creating Company. Harvard Business Review, 69, 96-114.

Organización de Cooperación y Desarrollo Económico (OCDE). (1999). Managing National Innovation Systems. París: OCDE.

Porter, M. (1980). Competitive Strategy: Techniques for analyzing industries and competitor. New York: Free Press.
Rallet, A. y Torre, A. (2004). Proximité et localization. Économie Rurale, 280, 103-129.

Sanz, F. J., Rodríguez-Zúñiga, M. y Mili, S. (1997). El sector del Aceite de Oliva ante la globalización de mercados: reflexiones desde una perspectiva de demanda. Economía Agraria, 181, 209-242.

Sanz, F. J., Rodríguez-Zúñiga, R. y Mili, S. (1998). Estrategias competitivas ante la globalización de los intercambios comerciales: la cadena del aceite de oliva. Revista Agroalimentaria, 4(7), 109-120.

Spear, R. (2001). The Cooperative Advantage. Annals of Public and Cooperative Economics, 71, 507-523.

Suárez, I. (1999). Decisiones de recorte de plantilla en la gran empresa española. Investigaciones Europeas de Dirección y Economía de la Empresa, 5, 79-100.

Vargas-Montoya, P. (2000). Características de los activos intangibles. Barcelona: Fundación Iberoamericana del Conocimiento. Disponible en: http://www.unipamplona.edu.co/unipamplona/portalIG/home_10/recursos/ general/documentos/pdf/14072011/1 caracteristicas. pdf 